\title{
NEVER TAKE TEAMWORK FOR GRANTED! A TUTORSHIP EXPERIENCE ON SOFT-SKILLS
}

\author{
Francesca MATTIOLI and Silvia FERRARIS \\ Politecnico di Milano
}

\begin{abstract}
Collaborative design-based learning is widely employed within academic design education, being recognised as a valuable pedagogical approach historically rooted and yet well-established within the design studio pedagogy. However, this great learning potential may not consolidate if it is not well integrated into the didactics. From this initial observation in the context of the MSc Design \& Engineering course at Politecnico di Milano the researchers developed a tutorship path implemented for a group of students along their entire master's programme, both in presence and remotely. The paper presents this action research which was conducted through the intervention and support of students in different key moments of their training. The tutorship on teamwork has been focused on providing activities to effectively perform initial team agreement, mid-term, and final evaluations (i.e., self, peer, and team assessments), along with constant support and coaching on team management. All these activities have been designed and provided in parallel to the design studios of the master's, meaning that each of them was related to real situations occurring during students' collaborative work. Qualitative data has been collected through a focus group organised with a group of 6 students at the end of the programme to evaluate the proposed path on teamwork. Four relevant themes emerged from data interpretation, which could inform teachers in the design of formalised paths on teamwork and soft skills. Additionally, the research contributes to wider discussion on soft skills development, with particular regards to teamwork.
\end{abstract}

Keywords: Culturally plural teams, design education, design-based learning, tutorship on teamwork

\section{INTRODUCTION}

Collaborative design-based learning is widely employed within academic design education, being recognised as a valuable pedagogical approach historically rooted and yet well-established within the design studio pedagogy. In numerous design schools, students - working in small groups - learn to design by designing together. With this setting, didactics provide a space for students to develop both hard skills related to design and soft skills related to more transversal domains such as collaboration, communication, work organisation just to mention a few. However, this great learning potential may not consolidate if it is not well integrated into the didactics. According to Savin-Baden and Howell (p.78) "few students come to higher education with well-developed team skills and to function in teams, they will need a range of skills and abilities that include interpersonal skills, active learning, team building and management, inquiry skills, conflict skills and presentation skills" [1]. Indeed, if it is assumed that students are already trained or prepared to work in teams, it could happen that they will work in teams without having the right set of skills and proper tools. In our context of Politecnico di Milano we observed that from the second year of bachelor until the final year of master's students are engaged with teamwork in the majority of design-based learning courses which are minimum one per semester. Despite the massive employment of collaborative learning a structured training on teamwork and collaborative skills is missing. As a result, students have to deal with the uneasy sides of collaboration by themselves (e.g., diversity, disagreements, different backgrounds, different abilities), getting frustrated and overcoming the issues not always in the most effective, sustainable, or inclusive ways. In this regard the role of what we defined as tutor could play a crucial role, creating a space of dialogue and reflection on the collaborations. Using facilitation [2] or coaching [3] [4], staff members could contribute to supporting raising awareness on effective approaches to teamwork. In the context of the Master's of Science in Design \& Engineering (D\&E), an interdisciplinary and highly international course, the researchers developed a dedicated path, called Tutorship for Teams (T4T). Even if the path was initially conceived to dedicate time to raise students' awareness on the importance of cultural 
sensitivity in plural teams, it became more of a basic training and tutorship on teamwork, since the majority of students never received a formal training before. T4T is the first iteration of a path lasting the whole duration of the master's programme and entirely dedicated to teamwork. The paper presents the way T4T was implemented and evaluated. Qualitative data collected through a focus group with students are reported and interpreted to disclose the recurring themes emerging from participants' conversation.

\section{METHOD}

\subsection{Tutorship for Teams (T4T): a transversal path dedicated to teamwork}

As part of the teaching staff, the researchers structured a tutorship path in the context of D\&E at Politecnico di Milano for a group of students along their entire master's programme between 2019 and 2021. T4T included activities addressed to different groups of students of D\&E design-based learning courses. 51 students participated in all the activities proposed in the path. The fundamental idea underpinning the T4T design was to provide tutorship for students engaged in teamwork in the Design Studios, that were planned during the first three semester of the two-years master's. These semesters are dedicated to courses, while in the fourth semester students must carry out their internship and work on their thesis project. The activities proposed in the T4T were divided into two semesters (i.e., the first and the third) and developed in parallel to the Design Studio of those semesters (i.e., Product Development Design Studio 1 and the Final Project Work).

\subsubsection{T4T first part: Seminar on Teamwork in the Product Development Design Studio 1}

A seminar on teamwork was proposed to the students during the first semester of D\&E, going from September 2019 to January 2020. This first activity was optative and opened to all the students enrolled in the Product Development Design Studio 1, that were divided in three parallel courses with different teachers. The professors of the sections presented the seminar on teamwork as an optative activity and one section decided to push student's participation by considering it in their final assessment. The initial activity was organised in October, after teams were formed, and there was a general presentation with the students (around 100) of the tutorship path for the first semester. The presentation, organised and done by the tutor (Author 1) was aimed at presenting the reasons and the objectives behind the whole path and introducing the scheduled activities. Then the second half of the meeting was dedicated to two activities 1) self-evaluation using the CATME scale [5] [6] and 2) the creation of a team agreement done collectively by each team and containing rules, roles, individual expectations, and contributions of all team members. During the first meeting the email contact of the tutor was shared with students, as the person to be contacted if a support on teamwork was needed. The second optative activity consisted in a private group review about teamwork with the tutor; teams of students had to autonomously contact her and set an appointment between November and December. Not being part of the teaching staff of the courses the tutor support was intended as external viewpoint and support. It was several times clarified by both teachers and the tutor that discussions during the teamwork reviews would remain private between the tutor and the group and would in no way be communicated to the course staff. This decision was driven by the context since the local education system is quite strongly hierarchal and therefore teachers decided not to directly interfere with teams' dynamics. During the 1-hour review, three main activities were proposed 1) individual self-evaluation using the CATME scale, 2) sharing of individual perceptions on teamwork and 3) the discussion and adjustment of the team agreement done collectively by all team members and the tutor. The participation to this second activity was modest.

\subsubsection{T4T second part: Teamwork Module in the Final Project Work}

The second part of the T4T was proposed only to the students in one of the sections during their third semester of D\&E, approximately from September 2020 to January 2021. In this case the activities were proposed in the form of a mandatory Teamwork Module in the Final Project Work course, a designbased learning course where the tutor is part of the teaching staff. Due to the pandemic emergency, the course was blended until mid-October when, due to local restrictions, it became completely remote together with teamwork. The Module on Teamwork consisted of three main steps; the first was organised in September 2020 after the teams' formation. After a brief presentation of the module each team was required to create its team agreement. Then the second step was organised after the mid-term presentation in early November and in this occasion, students performed a self-, peer and group evaluation using the Teamwork Colour Matrix tool [7] and presented it to the rest of the team. The aim 
of the activity was guaranteeing a moment of reflection for all the students and for each team to discuss and improve its organisation. After this step all the team had a review with the tutor which was completely dedicated to teamwork. The last step took place during the Christmas break, immediately before the final presentation, and it was nearly identical to the second step. The only difference was that students were required to deliver a final one-page report on teamwork. Based on the whole module, the tutor assessed the learning trajectory of each team and the soft skills acquisition demonstrated by the team throughout the course. This assessment was composed of a formative assessment, that was sent to each team in the form of a feedback, and a summative assessment, which was incorporated into the average of the grades of all teaching staff.

\subsection{Evaluating the experience: a focus group with students}

Qualitative data has been collected through a focus group organised with students at the end of the tutorship. The main objective behind the data collection was to highlight positive elements and critical points in regard to the tutorship proposed, to collect suggestions and, most importantly, to collect their impressions, perceptions and point of views on the activities proposed. At the end of the Final Project Work the researchers sent an open call to the 51 students that experienced the whole tutorship path, asking for volunteers willing to discuss their experience by participating in the focus group. People willing to participate had to fill an online form declaring their interest: 11 students answered the form and 6 were selected for the focus group. The participants were both males $(n=4)$ and females $(n=2)$, coming from 5 different groups of the Final Project Work course, all Italians and with a bachelor degree in industrial design awarded in an Italian University, which was Politecnico di Milano for the majority of them $(n=4)$. Focus group was based on methods described by Cardano [8] and organised online. A significant modification was to have only one moderator conducting the focus group, being also the principal proponent and the T4T tutor. The choice of having only one moderator was determined by the need to ensure students freedom of expression while telling their experiences and comments about the T4T path. Due to the trustful and direct relationship built between the students and the tutor throughout the T4T, the decision was to avoid any possible uncomfortable situation for the students such as having a professor or an unknown person listening to the discussion.

Table 1. Focus group discussion guide

\begin{tabular}{|l|l|}
\hline \multicolumn{1}{|c|}{ Items to be discussed } & \multicolumn{1}{c|}{ Questions } \\
\hline 1. Individual overall impression & Which is an image that represents the proposed tutorship path on teamwork? \\
\hline 2. Positive elements & What were the three most positive elements? \\
\hline 3. Critical elements & What have been the main critical points? \\
\hline 4. Expectations & What you would have liked but did not receive? \\
\hline 5. Suggestions & Do you have any suggestions for improving the path? \\
\hline
\end{tabular}

The focus group was composed by three main parts 1) an introduction by the moderator presenting the research and recapping the steps of the T4T path; 2) a guided discussion of around two and a half hours and 3) a conclusion by the moderator. The guided discussion, where the data collection took place, was organised as follow:

- $\quad$ the moderator posed the first question following the discussion guide (tab.1).

- $\quad$ left some minutes for the student to reflect and formulate their own answers.

- $\quad$ each participant was invited to respond to the question when she felt like it.

- $\quad$ after each person answered some more minutes were provided for all to add further comments emerged by listening to others.

- $\quad$ the moderator posed the next question and so on and so forth.

The whole discussion was monitored, and video recorded; the moderator took notes during the focus group and transcribed the records afterwards. The transcripts were then systematically reviewed; each segment of conversation was labelled in accordance with its emerging themes [9] and all labels analysed and clustered into four most recurring themes, described in the following section. 


\section{RESULTS: EMERGING THEMES}

\subsection{Generalised lack of formalised culture around teamwork}

A theme that strongly emerged from the dialogue is the recognition of a lack of formalised culture around teamwork. Indeed, students are very much accustomed to teamwork from the first years of bachelor, but they had never been formally trained to it.

...we should be used to talking about groups, it should be almost natural. In the sense that certain dynamics should have been dealt with even before the master's degree because we did two design studios a year, so there was no lack of opportunities. When I got to the fourth year, I personally realised how naive I was, because the project came out of the group, and to have never spoken, except in some very critical situation perhaps when everything was almost in shambles and you had to talk, is almost absurd.

The acknowledgement of this lack of culture, is also pointed as one of the main causes of the initial mistrust, low engagement, low participation to the T4T path. From the conversation emerges that students initially participated only because they wanted to please the teachers, especially when they understood that this activity would have been positively evaluated by professors.

It's better if we do it because apparently this will be considered positively by the teachers and it doesn't cost us too much, let's do it but if it were up to us, we wouldn't have done it.

Despite having work in teams for years, it emerges that students in their final years perceive themselves as "highly experienced on teamwork" and therefore initially underestimated the T4T path as a fruitful learning experience.

We treated it more as a "fill in a questionnaire" activity as if we were in junior high or high school, only later we took it a bit more seriously.

Interestingly, by participating in T4T students realised they were not used to talking about teamwork and saw it as something that can be discussed, questioned, and improved. In their view the activity proposed supported the teambuilding because making rules and roles explicit (i.e., team agreement) guaranteed higher accountability of teammates. On the other hand, the structured moments for sharing their individual perceptions about teamwork and teammates were considered awareness-rising, contributing to achieve better team results, to improve teams' dynamics and communication.

I connect directly to this last thing that GL said, the awareness was one of my positive points [ndr in evaluating the path]. To start talking about teamwork, I think it is the most important part of the path.

\subsection{Collaborative skills as a basic knowledge}

Despite the lack of formalised culture and knowledge around teamwork, students referred to collaborative skills as basic knowledge for designers and, most of all, for design students.

As designers, as people who work in groups all the time, the group world should be open to us, it should be something extremely natural, which it is not at the moment. We will probably talk about groups in a mature way in a few more years, because we are late.

The excerpt opens another sub-theme strictly related to the concept of teamwork as basic knowledge, namely the fact that T4T path should have been present within bachelor's programme in design. Throughout the conversation, participants repeatedly mentioned the need to train students and provide basic tools for teamwork starting from the bachelor's programme.

Going back to a point we discussed earlier, I know that [ndr in D\&E] it was done for the master's degree because it is more international, but I would have already introduced this method of team working at the bachelor's degree.

All the participants suggested to propose some type of structured activities on teamwork starting from the first years of designers training, because the current situation is that students learn to manage teams without guidance and therefore not necessarily in the most adequate way.

Even though we had been working in a group for five years now, it was always our own thing within the group, and everyone developed their own ways of managing it. In this way [ndr with T4T], just by talking about it, by the fact that teamwork emerges as a situation, as a discussion, it just gives you awareness and makes you say "ok, maybe there are more structured and functional methods to work in groups" [...] So just having done a course like this opens your mind for many aspects, for sure.

Another interesting element identified by students is that, in their experience, teamwork and collaborative skills were considered fundamental knowledges in other contexts such as in the work field 
(e.g., companies, career service of Politecnico di Milano) or in their extracurricular activities (e.g., theatre courses, circus school).

I wanted to comment on the fact, as FF already said, that in our university courses there were none on teamwork before. And I totally agree! I've already had experience in doing activities of this kind when I took part in workshops with companies [...] Not having done courses of this kind before at university is paradoxical, it's strange, at least in our course, since in any case in design we will always work in teams.

\subsection{Engage students: mandatory vs optative activities}

On several occasions, students have questioned and expressed opinions on the most appropriate way to deliver teamwork courses or paths: a recurring theme concerns the comparison between compulsory or optative activities. The group did not agree on a single opinion, but their experience in T4T provides an understanding of advantages and disadvantages of both modalities. For the first optional part of T4T, students demonstrated lower engagement with the proposed activities because they saw them as "another thing to add to the list of things to do". They really did not understand the support they could have gained from the path, but rather participated, as previously mentioned, to "please the teachers" in the hope their participation would have considered as a plus in the final assessment of the design studio. In this regard, what we labelled as "cornering" from teachers' side (i.e., rising the final grade for those that participated in the T4T) emerged as a controversial aspect in students' experience. From one side students criticised when the teachers applied gimmicks.

The professors had told us "this activity will then be considered in the final assessment" and I remember [...] my group didn't really like that.

On the other side because of this "cornering" students decided to participate in the T4T activities, demonstrating that in an assessment-driven model of education this could be an effective.

The first year our group did it under pressure from the teacher because she said she would give us an extra grade if we did it. The only reason, honestly, the only reason why we did it.

Indeed, in the second part of T4T, when the activity was integrated in the course and mandatory for all, the students participated and appreciated the majority of activity proposed.

I really liked this activity because of the clarity it gave me and the group, especially in the second year, where it was no longer optional, but part of the course.

Paradoxically I preferred this year where it was a part of the course, it was a compulsory part, but we didn't even feel it too much because it had to be done.

After several contribution on this theme, one insightful idea emerged.

The fact that it's optional gives you a bit of an impression that it's not key to the project, to the group, and so on. Utopianly, it should be optional in my opinion. The willingness to participate should be something that comes from the group itself. And again, here I come back to the fact that this is totally our fault [...] If at our age we need an activity to be compulsory in order to do it, then something is wrong. Obviously from a practical point of view [ndr a mandatory activity] is the only thing possible, because if it was only optional, we wouldn't have participated and therefore we wouldn't even be here talking about it probably.

\subsection{The role of the tutor}

The role of the tutor and the students-tutor relationship appear to be a crucial factor in the success of T4T. From students' perspectives, a key turning point for the path was the moment when they understood they could trust the tutor, as a person willing to listen to them, to help them in solving their team issues and to support them in improving teamwork.

It's good to have an impartial person, who can listen to you, ask you questions, let you open up. We understood the fact that you were impartial, but after a while... it took us a while... I have to tell you, in the first year first semester we still thought you were a bit of a professor, your role wasn't very clear [...] then I opened up a lot more and I think my group mates were a lot more relaxed to talk.

Another interesting fact is that students appreciated the tutor being an "external-internal" person. In their view the tutor should be seen as a person not involved in the assessment but knowledgeable about their projects and course dynamics.

In my opinion it is fundamental that this role is covered by someone internal-external, someone who knows what happens in the course, who is present in the course, who knows for example what happens in your relationship with the professors but who is not a professor, someone who does not evaluate you.

Moreover, to build this trust, it was crucial that the tutor had a direct experience in the course as a student.

The thing you said at the beginning when you introduced yourself is "I come from your experience, I've been through what you've been through, I'm like you with a bit more experience, I'm a person who seeks 
dialogue with you, I know what you're going through and what you're going to face". So, you became an authoritative figure as a result of the experience you had [...]

All the students denied the possibility to be evaluated on the teamwork and soft skills component of their learning experiences in design studio. This is particularly interesting because no one considered that this already happened in the Final Project Work, where the tutor explicitly evaluated teams on this component.

\section{Discussion}

The engagement with the focus group discussion was very high and all the participants contributed to it, also building their reflection on the contributions of others. The existing trustful relationship between the moderator (i.e., tutor of T4T) and the participants contributed to a calm, sincere and constructive atmosphere. The main contribution of this paper is to provide students' viewpoint on teamwork in design education at the end of their academic career and to address their perception on the implementation of tutorship path for teams within design-based courses. The overall evaluation of path was positive, even though students' engagement within T4T was initially low due to unclear understanding of the general aim behind the proposed activities. After the initial distrust, the activities acted as an awareness-raising factor, since it was the first time for all that a formal training path on teamwork was offered within an academic context. The four relevant themes emerged, revealed that much more could be done to improve formal training on teamwork. The main limit of the research is that the emerging themes should be extended and validated through further focus groups and in-depth interviews with students, possibly with reference to multiple institutional contexts. Beyond that, the described themes should be considered as a starting point to open some relevant debates that future research should address to foster the formalisation of training paths dedicated to teamwork in design institution that employ the collaborative learning approach. In our view, one of the most important results of this research is that the role of teamwork tutor emerges as a key figure within the path: further studies could provide deeper understanding of the characteristics and possible training for this role.

\section{REFERENCES}

[1] Savin-Baden M. and Howell Major C. Foundations of Problem-based Learning, 2004 (McGrawHill Education, UK).

[2] Savin-Baden Maggi. Facilitating problem-based learning, 2003 (McGraw-Hill Education, UK).

[3] van Diggelen M. R., Doulougeri K. I., Gomez-Puente S. M., Bombaerts G., Dirkx K. J. H., and Kamp R. J. A. Coaching in design-based learning: a grounded theory approach to create a theoretical model and practical propositions. International Journal of Technology and Design Education, 2019.

[4] Flipsen B. and Persaud S. Handle with care: Coaching multi-diverse project groups to become healthy design teams. In Proceedings of the 22nd International Conference on Engineering and Product Design Education, E\&PDE 2020, September 2020.

[5] Ohland M. W., Loughry M. L., Woehr D. J., Bullard L. G., Felder R. M., Finelli C. J., and Schmucker D. G. The comprehensive assessment of team member effectiveness: Development of a behaviourally anchored rating scale for self- and peer evaluation. Academy of Management Learning and Education, 2012, 11(4), pp. 609-630.

[6] Mattioli F. and Ferraris S. Self-evaluation and peer evaluation tool for design and engineering teams: experiment conducted in a design studio. In Towards a New Innovation Landscape: Proceedings of the 21 st International Conference on Engineering and Product Design Education, Department of Design, Manufacturing and Engineering Management, University of Strathclyde, UK, 12th 13th September 2019, pp. 364-369.

[7] Calvo Ivanovic I. and Mattioli F. A colour is worth a thousand words! A colour-based tool to foster communication in culturally-plural teams. In Y. A. Griber and V. M. Schindler (Eds.), The International Scientific Conference of the Colour Society of Russia: Selected papers, 2021, pp. 125-130 (Smolensk State University Press).

[8] Cardano M. La ricerca qualitativa, 2001 (Il Mulino, Bologna).

[9] Lune H. and Berg B. L. Methods for the Social Sciences Global Edition, 2017. 